\title{
Double-stage H-Darrieus wind turbine - rotor aerodynamics
}

\begin{abstract}
H-Darrieus wind turbines, due to their simple design and relatively low manufacturing costs have recently received much attention particularly for standalone applications. However startup issues associated with their operation restricted their operation in areas of low average wind speed and encourages engineers to develop novel design. Several design proposed in this way but in most cases design came up with complex sensing mechanisms and mechanical actuators or high cost manufacturing parts. A recent rotor design called double Darrieus rotor proposed as a German patent case bridged these complexities appropriately. The aim of present study is to investigate this innovative design from aerodynamic point of view by means of validated CFD techniques. A flow-driven simulation setup based on 6DOF calculations employed in order to study rotor operation from stand still until peak performance obtained. Results from these precise modeling reveal the superiority of the proposed double-stage design in compare with the original H-Darrieus rotors in terms of start-up behavior and optimum performance.
\end{abstract}

Keyword: CFD; Double-stage Darrieus rotor; H-Darrieus; Rotor aerodynamics; Unsteady simulation 